\title{
Triagem para distúrbios visuais em escolares no município de Campo Grande, Mato Grosso do Sul, Brasil
}

\author{
Screening for visual disturbances in schoolchildren in the city of Campo Grande, \\ Mato Grosso do Sul, Brazil
}

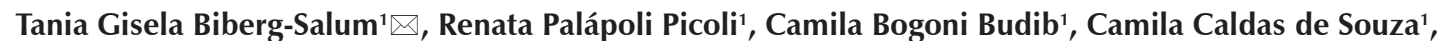 \\ Carlos Magno Guimarães' ${ }^{1}$ Eduardo Silva Aguiar'1, Livia Oliveira Cunha' ${ }^{1}$, Marco Antônio Kawabata ${ }^{1}$, \\ Matheus Ribeiro Comparin', Wagner Luiz Engelman'
}

${ }^{1}$ Curso de Medicina da Universidade Anhanguera-Uniderp. Campo Grande, MS.

\section{RESUMO}

Objetivos: Verificar a ocorrência de alterações visuais em escolares de uma instituição filantrópica do município de Campo Grande, Mato Grosso do Sul, Brasil.

Métodos: Foi realizado um estudo transversal com escolares de seis a 12 anos de idade, de ambos os sexos, que frequentavam uma instituição filantrópica no ano de 2012. Para a triagem visual foi utilizado o teste de Ishihara e a leitura da escala optométrica de Snellen. Posteriormente, os escolares com resultado alterado foram referenciados para avaliação com especialista em oftalmologia.

Resultados: Foram incluídos no estudo 94 escolares, entre os quais 18 apresentaram alteração na triagem visual, sendo cinco com suspeita de discromatopsia e 13 com baixa acuidade visual segundo a Escala de Snellen. Onze escolares compareceram para consulta com especialista, sendo três por suspeita de discromatopsia e o oito por alteração da acuidade visual. Após a avaliação oftalmológica, verificou-se que, dos escolares com suspeita de discromatopsia, um apresentou dúvida quanto ao diagnóstico positivo. No tocante aos escolares triados por alteração da acuidade visual, dois apresentaram déficit visual.

Conclusões: Os resultados demonstraram que embora uma parcela importante de escolares apresentasse alteração visual na triagem, a alteração não foi confirmada na maioria das avaliações oftalmológicas. Estes achados salientam a importância da triagem para detectar alterações oculares, desde que seguida por avaliações oftalmológicas, para o correto diagnóstico e orientação.

DESCRITORES: saúde escolar; acuidade visual; transtornos da visão.

\section{ABSTRACT}

Aims: To investigate the occurrence of visual disturbances in schoolchildren from a philanthropic institution in the city of Campo Grande, Mato Grosso do Sul, Brazil.

Methods: A cross-sectional study was conducted with students aged six to 12 years, both male and female, who attended a philanthropic institution in 2012. The Ishihara test and the Snellen chart were used for visual screening. Later, those students with abnormal results were referred to a specialist.

Results: Ninety-four schoolchildren were included in the study. Eighteen were found by the visual screening to have some impairment: suspected dyschromatopsia in five and poor visual acuity in 13, according to the Snellen chart. Eleven students, three with suspected dyschromatopsia and eight with poor visual acuity, went to see a specialist. After ophthalmologic evaluation, one child with suspected dyschromatopsia was unsure about the positive diagnosis. Among the students with abnormal visual acuity, two presented with visual deficit.

Conclusions: The results show that, although a considerable number of students presented with some type of visual problem during the visual screening, impairment was not confirmed by most of the ophthalmologic evaluations. These findings highlight the importance of screening for the detection of visual disorders, provided that it is followed by ophthalmologic assessments for proper diagnosis and guidance.

KEY WORDS: school health; visual acuity; vision disorders. 
Abreviaturas: CEMED, Centro de Especialidades Médicas da Uniderp; IBGE, Instituto Brasileiro de Geografia e Estatística; Uniderp, Universidade para o Desenvolvimento do Estado e da Região do Pantanal.

\section{INTRODUÇÃO}

O comprometimento da saúde ocular na infância representa importante inibidor do desenvolvimento da criança, com potencial para provocar sequelas na sua vida adulta. Destaca-se que a deficiência visual é raramente percebida pelos pais e familiares antes do início da vida escolar, porque no ambiente doméstico a criança não tem percepção do seu comprometimento [1].

Pesquisas apresentam que grande número de crianças em idade escolar nunca teve acesso a um exame oftalmológico especializado [2] e, de acordo com dados publicados pelo Conselho Brasileiro de Oftalmologia em 2015, o número potencial de crianças em idade escolar (cinco a 15 anos) com problemas de erros de refração é de 15 milhões, sem considerar as demais causas de diminuição da acuidade visual. É importante ressaltar que, dentre as causas de mais da metade das crianças que ficam cegas, no mundo, estas poderiam ser evitáveis (tratáveis ou preveníveis) [3].

A visão é um dos fatores mais importantes no processo de ensino-aprendizagem. Crianças e adolescentes com alteração da acuidade visual, muitas vezes, não apresentam rendimento escolar satisfatório [4]. Em assim sendo, ações de promoção em saúde e prevenção de agravos em ambiente escolar devem ser desenvolvidas em parceria com serviços de saúde, instituições de ensino superior e comunidade, para que o atendimento aos escolares possa ser ampliado e promova a saúde em todas as suas dimensões colaborando assim, inclusive, para o melhor desenvolvimento do aprendizado.

É válido salientar que, no Brasil, a percepção sobre o conceito e a prática de saúde escolar e de promoção da saúde tem mudado. Essas mudanças têm sido implantadas por meio de políticas públicas voltadas para a saúde do escolar [5]. Dentre estas novas perspectivas, tem sido recomendada a realização da triagem da acuidade visual entre os escolares para se identificarem, precocemente, as alterações que podem dificultar os seus processos de aprendizagem. Tais alterações podem se manifestar como problemas de leitura e/ou da escrita e, consequentemente, baixo rendimento escolar. Assim, a escola é um espaço privilegiado para a implementação de políticas, uma vez que $96 \%$ das crianças e dos adolescentes a frequentam [6].

Considerando a importância da parceria entre entidades escolares e equipes de saúde das Unidades Básicas de Saúde da Família, os acadêmicos do Curso de Medicina da Universidade Uniderp desenvolveram este estudo com o propósito de verificar a ocorrência de distúrbios visuais em escolares dessa instituição, a fim de tentar corrigir as possíveis alterações encontradas, para assim, reduzir os impactos da privação visual no desenvolvimento cognitivo das crianças.

\section{MÉTODOS}

Realizou-se um estudo do tipo transversal em uma instituição filantrópica da cidade de Campo Grande, Estado de Mato Grosso do Sul, no período de agosto de 2011 a agosto de 2012. Foram convidadas a participar da pesquisa 100 crianças, de ambos os sexos, com idades entre seis e 12 anos, que frequentavam regularmente a instituição no ano de 2012. Os escolares estavam matriculados no ensino fundamental, do segundo ao oitavo ano, em escolas públicas do município. Foram incluídas no estudo as crianças e adolescentes cujos responsáveis concordaram em participar do estudo e assinaram o Termo de Consentimento Livre e Esclarecido, tendo os escolares assinado o Termo de Assentimento para Menores. Foram excluídos do estudo os estudantes que não apresentaram ambos os termos assinados ou que não se apresentaram na instituição nos dias definidos para a triagem. Ao final, a partir dos critérios estabelecidos, a amostra foi composta por 94 sujeitos.

Para a triagem visual, no que concerne à avaliação para as cores, optou-se pela utilização do teste de Ishihara, editado em 1974, constituído por 17 pranchas pseudoisocromáticas para a identificação do distúrbio de percepção das cores em escolares alfabetizados. O teste de triagem foi realizado pelos acadêmicos pesquisadores, colocando-se as figuras a $75 \mathrm{~cm}$ do paciente, em ângulo reto com o eixo visual, sob iluminação natural, como determina o manual de instruções do livro. Os escolares foram avaliados individualmente, em sala privativa, para manter o sigilo quanto ao resultado. A triagem concernente à acuidade visual foi medida em um olho de cada vez, sendo um dos olhos ocluído com o auxílio de um oclusor externo. Por meio da leitura da escala optométrica de Snellen, o teste foi realizado pelos acadêmicos após treinamento com oftalmologista. A criança foi posicionada a 5 metros da tabela e lhe foi solicitado que identifi- 
casse os signos dispostos na mesma. Para as crianças com dificuldades de alfabetização foi utilizado o optótipo de "E" de Snellen, tendo a criança que indicar a posição da letra "E". Para os alfabetizados foi utilizada tabela de letras, que apresenta uma ordem decrescente quanto ao tamanho das mesmas. Iniciou-se o exame pelos optótipos de maior tamanho e seguiu-se em ordem decrescente conforme o acerto do examinado. Os acadêmicos anotaram o valor equivalente à última linha lida sem dificuldade, o que traduz a melhor acuidade visual alcançada em cada olho. As crianças em uso de óculos foram testadas com e sem as lentes corretivas [7]. Aquelas que apresentaram acuidade visual $\leq 0,7$ foram encaminhadas ao especialista para confirmação diagnóstica e conduta pertinente.

Após a triagem, os resultados dos exames foram impressos em duas vias, sendo uma entregue aos pais e/ou responsáveis. Os escolares com suspeita de alterações foram referenciados para o Centro de Especialidades Médicas da Universidade Uniderp (CEMED), para avaliação com oftalmologista. Posteriormente, conforme a necessidade, houve encaminhamentos para o setor de nível terciário da Saúde Municipal, por meio da diretoria da unidade básica de saúde da família Nossa Senhora das Graças.

Foram respeitados os critérios éticos conforme a Resolução 466 de 2012 do Conselho Nacional de Saúde e o estudo foi aprovado pelo Comitê de Ética em Pesquisa da Universidade Uniderp, conforme parecer no $153 / 2010$.

Para a organização dos dados utilizou-se o programa Microsoft Office Excel 2007, sendo os resultados apresentados em forma de tabelas, analisados por estatística descritiva com frequência absoluta e porcentagem, e discutidos de acordo com a literatura pertinente.

\section{RESULTADOS}

Foram triados 94 escolares, sendo 52 do sexo masculino. Em relação à idade, a mínima foi de seis anos, a máxima de 12 anos, a média de nove anos e a mediana de 11 anos. Na triagem dos escolares, realizada pelos acadêmicos, verificou-se a suspeita de alteração visual em 18 (19,1\%) crianças. Destas, cinco (5,3\%) apresentaram suspeita de discromatopsia e $13(13,8 \%)$ de baixa acuidade visual (Figura 1).

No que diz respeito à discromatopsia, dos cinco escolares com alteração na triagem, três eram do sexo masculino e dois do sexo feminino. Já na avaliação pela Escala de Snellen, dos 13 estudantes que apresentaram alterações, oito eram do sexo masculino e

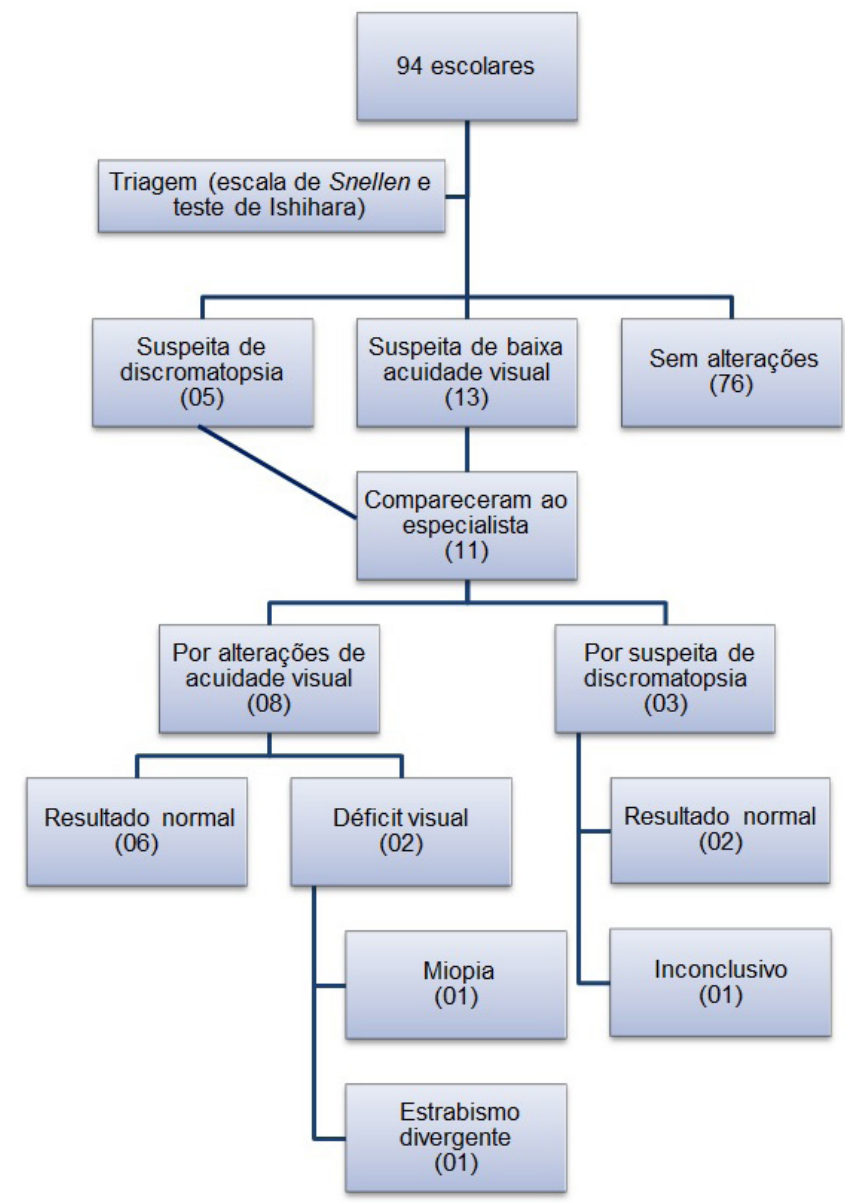

Figura 1. Triagem para alterações visuais e avaliações oftalmológicas realizadas em 94 estudantes em Campo Grande, Mato Grosso do Sul, Brasil, 2012.

cinco do sexo feminino. Dos 13 escolares nos quais foi observada baixa acuidade visual, quatro $(30,8 \%)$ apresentaram alterações em ambos os olhos e nove em apenas um. Em um escolar foi percebida perda total de visão no olho esquerdo.

Após a realização das triagens visuais, todos os escolares que apresentaram algum grau de alteração foram encaminhados ao CEMED, para consulta com médicos oftalmologistas. Dos 18 escolares com triagem suspeita, 11 compareceram e foram avaliados pelo especialista, sendo que destes, três haviam sido encaminhados por suspeita de discromatopsia e oito por alterações na Escala de Snellen. Mediante a avaliação oftalmológica, verificou-se que dos escolares com suspeita de discromatopsia, dois apresentaram resultados normais e um apresentou dúvida quanto ao diagnóstico positivo. Para este último, foi recomendado retorno em um ano para novos exames.

No tocante aos escolares triados com alteração da acuidade visual, dos oito que compareceram dois apresentaram déficit visual, sendo ambos do sexo 
feminino; e seis obtiveram resultados normais. Os escolares diagnosticados com déficit visual na avaliação oftalmológica já faziam uso de lentes corretivas, sendo que um apresentava estrabismo divergente alternante e o outro, miopia. O primeiro foi referenciado pela Unidade Básica de Saúde da Família Nossa Senhora das Graças para acompanhamento em um serviço de nível terciário de saúde do município. Assim, a prevalência de miopia observada na amostra de 94 triados foi de $1,1 \%$.

\section{DISCUSSÃO}

A Escala de Snellen tem se mostrado um instrumento de avaliação bastante adequado para ser empregado em triagem para acuidade visual, por ser um teste de fácil aplicação, alta eficácia e baixo custo, não requerendo especialistas no seu manuseio, podendo contar com pessoal treinado sob supervisão. A facilidade de aplicação em grandes massas populacionais possibilitou sua utilização em diversos estudos com essa abrangência [8-10]. No entanto, em situações específicas, como nas deficiências visuais e/ou cognitivas, testes diferenciados podem ser necessários [11]. Em se tratando da avaliação da visão de cores, tem sido amplamente utilizado o teste de Ishihara, por ser este também de fácil aplicação [12]. É o mais utilizado mundialmente para a pesquisa de discromatopsias congênitas [13].

A faixa etária da amostra selecionada para este estudo foi mais ampla do que a analisada no estudo de Toledo et al. [9]; esses autores avaliaram apenas crianças de oito a 10 anos de idade. Considerase adequada a faixa etária investigada no presente estudo, por englobar a idade de sete anos, que, segundo alguns autores, é aquela em que o aparelho visual está completamente maturado, atingindo seu potencial máximo e ainda sendo capaz de melhora com intervenções precoces $[9,10]$.

A prevalência de alterações visuais encontrada no presente trabalho foi inferior em comparação ao estudo de Gianini et al. [14], que identificou $13,1 \%$ de baixa acuidade visual em escolares do primeiro ao quarto ano do ensino fundamental do município de Sorocaba.

De acordo com as informações fornecidas pelo Instituto Brasileiro de Geografia e Estatística (IBGE 2008) [15], aproximadamente $10 \%$ da população escolar tem problema visual. Estudo conduzido por Laignier et al. [10], com 168 crianças, evidenciou que $10(6 \%)$ apresentaram baixa acuidade visual na triagem. Destas, sete compareceram à consulta oftalmológica e cinco apresentaram diagnóstico de ametropia, sendo possível presumir que o procedimento da triagem foi realizado adequadamente.

No presente estudo, observou-se que $13,8 \%(n=13)$ dos escolares apresentaram resultados alterados na triagem realizada por meio da Escala de Snellen; destas, oito compareceram à consulta oftalmológica agendada e apenas duas tiveram a confirmação de déficit visual, o que demonstra a importância de confirmação diagnóstica pelo oftalmologista. Algumas hipóteses que podem justificar estes resultados envolvem falhas na execução da triagem da acuidade visual, como escolha e preparo do local para aplicação do teste, experiência dos examinadores na execução da triagem e orientação correta para as crianças responderem ao teste.

Dentre os 13 escolares com alteração na Escala de Snellen, a maioria relatou dificuldade para enxergar em apenas um dos olhos. No estudo de Laignier et al. [10], metade dos escolares apresentaram alteração em ambos os olhos e a outra metade em apenas um dos olhos.

No exame com o oftalmologista, obteve-se confirmação de resultado alterado em apenas dois escolares, sendo que um apresentou miopia e outro estrabismo divergente alternante. Oliveira et al. [8] encontraram que o erro refracional mais presente foi o astigmatismo hipermetrópico $(63,2 \%)$, enquanto Ribeiro et al. [17] relataram a hipermetropia fisiológica como a mais prevalente.

O número de escolares ausentes no dia da realização do teste com o especialista $(38,9 \%)$ deve ser considerado na discussão da prevalência de baixa acuidade visual. A elevada taxa de absenteísmo poderia ter relação de causalidade com a subestimação do resultado [14]. De acordo com estudo de Kara-José [18], essa ausência pode acontecer tanto pela resistência ou recusa dos pais em ter seus filhos examinados, quanto pela pouca compreensão pelos responsáveis do quanto a visão interfere no aprendizado dos seus filhos. Mesmo levando em conta que várias intercorrências pudessem impedir o comparecimento da criança na primeira data marcada para a avaliação pelo oftalmologista, mesmo com a remarcação da consulta, apenas $10 \%$ dos inicialmente faltosos compareceram. Isso pode ser considerado como uma dificuldade apresentada no presente estudo e um dos motivos da baixa prevalência de alterações encontrada.

Embora no presente estudo os aspectos socioeconômicos não tenham sido investigados, destaca-se que tais aspectos poderiam, indiretamente, influenciar no absenteísmo das crianças à consulta com o oftalmologista, conforme resultados obtidos por Adub 
Ottaiano [16], que ao investigarem os fatores que influenciaram no comparecimento dos escolares com suspeita de alterações visuais ao exame oftalmológico realizado após triagem, durante a Campanha Nacional de Reabilitaçao Visual "Olho no Olho", no município de Lins, observaram que os escolares com convênio médico privado apresentaram maior absenteísmo ao exame oftalmológico quando comparados aos que não tinham convênio médico privado.

Concluindo, os resultados demonstraram que embora uma parcela importante de escolares apresentasse alteração visual na triagem, a mesma não foi confirmada na maioria das avaliações oftalmológicas, pois dos 18 escolares com alteração na triagem, um apresentou resultado inconclusivo para discromatopsia e dois foram confirmados com déficit visual. Destes, um apresentava miopia e um estrabismo divergente alternante, o que demonstra a importância do diagnóstico e definição da conduta pelo especialista.

Percebe-se também que a continuidade da investigação da acuidade visual, por meio do comparecimento ao especialista, foi reduzida, o que reforça a necessidade de desenvolvimento de novas estratégias de sensibilização de pais e responsáveis quanto ao acompanhamento adequado da saúde ocular de seus filhos.

\section{AGRADECIMENTOS}

Agradecemos à Dra. Tereza Cristina Martins, que trabalha na Unidade Básica de Saúde da Família Nossa Senhora das Graças, que foi uma grande incentivadora do projeto, demonstrando interesse, eficiência e acrescentando muito nesta pesquisa.

\section{REFERÊNCIAS}

1. Gasparetto MERF, Temporini ER, Carvalho KMM, Kara-José N. Dificuldade visual em escolares: conhecimentos e ações de professores do ensino fundamental que atuam com alunos que apresentam visão subnormal. Arq Bras Oftalmol. 2004;67(1):65-71. http://dx.doi.org/10.1590/ S0004-27492004000100011

2. Silva CMF, Almeida DR, Bernardes RR, Bazzano FCO, Filho MM, Magalhães CHT, Atzingen DANCV. Desempenho escolar: interferência da acuidade visual. Rev Bras Oftalmol. 2013;72(3):168-71. http://dx.doi.org/10.1590/S0034-72802013000300005

3. Ávila M, Alves MR, Nishi M. As Condições de Saúde Ocular no Brasil. CBO - Conselho Brasileiro de Oftalmologia [Internet]. São Paulo; 2015 [cited 2015, Aug]. Available from: www.cbo.net.br/novo/publicacoes/Condicoes_saude_ocular_IV.pdf

4. Neto CAM, Moreira ATR; Moreira LB. Relação entre acuidade visual e condições de trabalho escolar em crianças de um colégio do ensino fundamental público de Curitiba. Rev Bras Oftalmol. 2014;73(4):216-9. http://dx.doi.org/10.5935/0034-7280.20140047

5. Brasil. Decreto no 6.286, de 5 de dezembro de 2007. Institui o Programa Saúde na Escola - PSE. Diário Oficial da União, Brasília, DF, 5 dez. 2007.

6. Dadalto EV, Nielsen CSCB, Oliveira EAM. Levantamento da prevalência de distúrbios da comunicação em escolares do ensino público fundamental da Cidade de Vila Velha/ES. Rev CEFAC. 2012;14(6):1115-21. http://dx.doi.org/10.1590/S1516-18462012005000024

7. Granzoto JA, Ostermann CSPE, Brum LF, Pereira PG, Granzoto T. Avaliação da acuidade visual em escolares da 1a série do ensino fundamental. Arq Bras Oftalmol. 2003;66:167-71. http://dx.doi.org/10.1590/S0004-27492003000200010

8. Oliveira CAS, Hisatomi KS, Leite CP, Schellini SA, Padovani CR, Padovani CRP. Erros de refração como causas de baixa visual em crianças da rede de escolas públicas da regional de Botucatu - SP. Arq Bras Oftalmol. 2009;72(2):194-8. http://dx.doi.org/10.1590/S000427492009000200012

9. Toledo CC, Paiva APG, Camilo GB, Maio MRS, Leite ICG, Gherra, MR. Detecção precoce de deficiência visual e sua relação com o rendimento escolar. Rev Ass Méd Bras. 2010;56(4):415-9. http://dx.doi.org/10.1590/S0104-42302010000400013

10. Laignier MR, Castro MA, Sá PSC. De olhos bem abertos: investigando acuidade visual em alunos de uma escola municipal de Vitória. Esc Anna Nery Rev Enferm. 2010;14(1):113-9. http://dx.doi.org/10.1590/S1414-81452010000100017

11. Batista CG, Nunes SS, Horino LE. Avaliação assistida de habilidades cognitivas em crianças com deficiência visual e com dificuldades de aprendizagem. Psicologia: Reflexão e Crítica. 2004;17(3):381-93. http://dx.doi.org/10.1590/s0102-79722004000300011

12. Urbano LCV. Discromatopsia: métodos de exame. Arq Bras Oftalmol. 978;41(5):236-52.

13. Martins GM, Bordaberry MF, Corrêa ZMS, Mânica MB, Costa JC, Telichevesky N, et al. Visão das cores em escolares: avaliação de um novo teste. J. Pediatr. 2001;77(4):327-30 http://dx.doi.org/10.1590/s0021-75572001000400016

14. Gianini RJ, Masi E, Coelho EC, Oréfice FR, Moraes RA. Prevalência de baixa acuidade visual em escolares da rede pública, Sorocaba. Rev Saúde Pública. 2004;38(2):201-8. http://dx.doi.org/10.1590/S0034-89102004000200008

15. Brasil. Ministério da Educação. Instituto Nacional de Pesquisas em Educação Básica (Inep). Censo Escolar da Educação Básica. [Internet]. Brasília; 2013 [cited 2015, Aug]. Available from: http://download.inep.gov.br/educacao_basica/censo_escolar/resumos_tecnicos/resumo_ tecnico_censo_educacao_basica_2013.pdf

16. Abud AB, Ottaiano JAA. Aspectos socioeconômicos que influenciam no comparecimento do exame oftalmológico de escolares com alterações visuais. Arq Bras Oftalmol. 2004;67(5):773-9. http://dx.doi.org/10.1590/S0004-27492004000500015

17. Ribeiro GB, Coelho ALD, Chaves PHP, Macedo RL, Silva TAB. Avaliação oftalmológica de crianças de escolas públicas de Belo Horizonte/ MG: um panorama acerca da baixa acuidade visual. Rev Bras Oftalmol. 2015;74(5):288-91. http://dx.doi.org/10.5935/0034-7280.20150059

18. Kara-José N. Importância da Correção Óptica nos Escolares [Internet]. Conselho Brasileiro de Oftalmologia; 2009 [cited 2015, Aug]. Available from: http://www.cbo.com.br/novo/medico/pdf/jo/ed122/6.pdf $\epsilon$ 